\title{
Helicobacter pylori and gastric carcinoma - from the view point of animal model
}

\author{
Toshio Fujioka, ${ }^{1}$ Kazunari Murakami, ${ }^{2}$ Masaaki Kodama, ${ }^{1}$ Jiro Kagawa, ${ }^{1}$ Tadayoshi Okimoto $^{2}$ and Ryugo Sato ${ }^{2}$ \\ ${ }^{1}$ Department of General Medicine, \\ ${ }^{2} 2^{\text {nd }}$ Department of Internal Medicine, Oita Medical University, Oita, Japan
}

\begin{abstract}
Many epidemiological studies have shown a strong association between chronic Helicobacter pylori infection and subsequent development of gastric carcinoma in humans. To confirm this link more clearly, it is necessary to use this bacterium in experimental studies to develop gastric carcinoma in suitable experimental animals. Persistent $H$. pylori infection has recently been achieved in the Japanese Monkeys and Mongolian gerbil models, with results demonstrating that the sequential histopathological changes in the gastric mucosa are closely mimic the gastric mucosal changes caused by $\boldsymbol{H}$. pylori infection in humans. Gastric mucosa infected with $H$. pylori exhibited significantly higher gastritis score, reduction in glandular height, increase in the number of $\mathrm{Ki}-67$ positive cells and over expression of p53 protein and p53 gene mutation in the Japanese Monkey Model. In the Mongolian gerbil model, $\boldsymbol{H}$. pylori infection enhances gastric carcinogenesis in combination with known carcinogens such as MNU and MNNG, and also demonstrated that $H$. pylori infection alone can result in the development of gastric carcinoma. However, diagnostic criteria of gastric carcinoma in animal models remain in the great discussion. These important results provide a starting point for further studies to clarify the mechanism of gastric carcinogenesis as a result of $H$. pylori infection and assist the planning of eradication therapy to prevent gastric carcinoma.
\end{abstract}

Key words: Helicobacter pylori, gastric carcinoma, animal model, Japanese Monkeys, Mongolian gerbils

\section{Introduction}

In June 1994, the International Agency for Research on Cancer (IARC), a branch of the World Health Organization (WHO), convened experts from 11 countries to examine the evidence linking a number of infectious agents with human cancer. Although there is inadequate evidence in experimental animals for carcinogenicity of infection with $H$. pylori, it was designated as a definite carcinogen (Group I) to the human stomach based on three prospective case-control studies ${ }^{1-3}$ reported in 1991.

However, the mechanisms by which $H$. pylori infection increases the risk of gastric carcinoma are currently unknown and the IARC emphasizes that there is inadequate evidence in experimental animals for carcinogenicity of infection with $H$. pylori ${ }^{4}$ In further studies attempting to reveal the possible mechanisms by which $H$. pylori contributes to the development of gastric carcinoma, many researchers worldwide have developed animal models of infection using Helicobacter species.

In our department, persistent $H$. pylori infection has recently been achieved in the Japanese Monkey and Mongolian gerbil model, with results demonstrating that the sequential histopathological changes in the gastric mucosa closely mimic the gastric mucosal changes caused by $H$. pylori infection in humans. ${ }^{5}$

\section{Development of the Japanese Monkey Model}

The use of monkey model provide us with the possibilities of long-term gastrofiberscopic follow up and repeated histological examinations of gastric mucosa using endoscopic biopsy specimens.

In our initial animal experiments, we endoscopically inoculated the $H$. pylori isolates from humans. The macroscopic gastritis induced by $H$. pylori was most prominent in the antrum and was milder from the body to the fornix of the stomach. Histologically, inflammatory cells were most intense in the lamina propria of the antrum.

Using these Japanese Monkey model, long term sequence of gastric mucosal changes such as gastritis scores, reduction of glandular height and an increase in

Reprint requests to: Dr. Toshio Fujioka, Department of General Medicine, Oita Medical University, Hasama-machi, Oita 879-5593, Japan, e-mail: fujioka@oita-med.ac.jp 
the number of immuno-positive cells for $\mathrm{Ki}-67^{6}$ and p53 proteins ${ }^{7}$ compared with the gastric mucosa of uninfected subjects.

In our recent study, we concluded that the appearance of p53 gene mutation ${ }^{8}$ may be closely associated with the degree of gastric mucosal atrophy, which depends on the duration of $H$. pylori infection.

However, we have not confirmed the occurrence of gastric carcinoma in the stomach of Japanese Monkeys.

\section{Development of the Mongolian Gerbil Model}

In 1996, the first study in Japan by Hirayama et al. ${ }^{9}$ reported that persistent $H$. pylori infection had been found in the glandular stomach of Mongolian gerbils (MGS/Sea; Seac Yoshitomi, Fukuoka, Japan), which had progressed to gastritis, gastric ulcer, and then to intestinal metaplasia. Honda et al. ${ }^{10}$ and Ikeno et al. ${ }^{11}$ subsequently confirmed similar sequences which demonstrated that these changes in the gastric mucosa were closely similar to those found in human gastric mucosa infected with $H$. pylori.

On the other hand, in 1998. Tatematsu et al. ${ }^{12}$ demonstrated that glandular stomach carcinomas developed in Mongolian gerbils to which nitroso compounds alone had been given. Specifically, it was confirmed that oral administration of the known carcinogen $\mathrm{N}$ methyl- $N$-nitrosourea (MNU) and $N$-methyl- $N$-nitro$N^{\prime}$-nitrosoguanidine (MNNG) resulted in the development of adenocarcinoma in the glandular stomach of $H$. pylori-sensitive Mongolian gerbils.
As a result of the successful development of gerbil models with $H$. pylori infection, several studies were reported from Japan, which agreed on a causal link between gastric carcinoma and $H$. pylori infection.

\section{Carcinogenicity of $\boldsymbol{H}$. pylori in Combination with Carcinogens}

Fox et al. ${ }^{13}$ reported an experimental study suggesting a possible carcinogenic role for Helicobacter species in the gastric mucosa after oral administration of MNNG in ferrets infected with Helicobacter mustelae. Ten ferrets were orally dosed with $50 \mathrm{mg} / \mathrm{kg}$ MNNG in olive oil. Nine of the ten ferrets developed adenocarcinoma. This was the first experimental study using carcinogens combined with infection with a Helicobacter species. However, a major problem with this study was that no uninfected ferrets were included as controls. Although spontaenous gastric adenocarcinomas have been reported ${ }^{14}$ in aged ferrets with $H$. mustelae even in the absence of carcinogen exposure, an important additional problem is that the bacterium used was not $H$. pylori but $H$. mustelae.

The results of three experimental studies recently conducted in Japan have confirmed that administration of MNNG or MNU to Mongolian gerbils with chronic $H$. pylori infection enhanced the development of different histopathological types of gastric carcinoma (Table 1). ${ }^{15-17}$

Sugiyama et al. ${ }^{15}$ reported the development of carcinoma in the gastric mucosa of Mongolian gerbils eval-

Table 1 Gastric Carcinogenesis in Helicobacter pylori-infected Mongolian Gerbils

\begin{tabular}{|c|c|c|c|c|c|}
\hline Author & Year & Strain & Study design (p.p.m) & $\begin{array}{l}\text { Incidence of } \\
\text { cancer }(\%)\end{array}$ & $\begin{array}{l}\text { Duration of } \\
\text { experiment (weeks) }\end{array}$ \\
\hline \multirow[t]{4}{*}{ Sugiyama et al. ${ }^{15}$} & 1998 & ATCC43504 & $\mathrm{HP} \rightarrow \mathrm{MNU}(10)$ & $7 / 19(36.8)$ & 40 \\
\hline & & & HP alone & $0 / 20(0)$ & 40 \\
\hline & & & $\mathrm{MNU}(30) \rightarrow \mathrm{HP}$ & $6 / 18(33.3)$ & 40 \\
\hline & & & MNU alone & $0 / 74(0)$ & 40 \\
\hline \multirow[t]{4}{*}{ Tokieda et al..$^{16}$} & 1999 & ATCC43504 & $\mathrm{HP} \rightarrow \mathrm{MNNG}(50)$ & $5 / 17(29.4)$ & 52 \\
\hline & & & persistant HP positive & $5 / 8(62.5)$ & 52 \\
\hline & & & HP eradicated & $0 / 9(0)$ & 52 \\
\hline & & & $\mathrm{Br} \rightarrow \mathrm{MNNG}(50)$ & $3 / 22(13.6)$ & 52 \\
\hline \multirow[t]{9}{*}{ Shimizu et al. ${ }^{17}$} & 1999 & ATCC43504 & $\mathrm{MNNG}(300) \rightarrow \mathrm{HP}$ & $12 / 27(44.4)$ & 50 \\
\hline & & & $\mathrm{MNNG}(300) \rightarrow \mathrm{Br}$ & $1 / 19(5.3)$ & 50 \\
\hline & & & MNNG(60) $\rightarrow$ HP & $6 / 25(24.0)$ & 50 \\
\hline & & & $\mathrm{MNNG}(60) \rightarrow \mathrm{Br}$ & $0 / 20(0)$ & 50 \\
\hline & & & $\mathrm{HP} \rightarrow \mathrm{MNNG}(100)$ & $4 / 27(14.8)$ & 50 \\
\hline & & & $\mathrm{Br} \rightarrow \mathrm{MNNG}(100)$ & $3 / 18(16.7)$ & 50 \\
\hline & & & $\mathrm{HP} \rightarrow \mathrm{MNNG}(20)$ & $12 / 25(60)$ & 50 \\
\hline & & & $\mathrm{Br} \rightarrow \mathrm{MNNG}(20)$ & $1 / 20(5)$ & 50 \\
\hline & & & HP alone & $0 / 20(0)$ & 50 \\
\hline
\end{tabular}

HP: Helicobacter pylori, MNU: $N$-Methy- $N$-nitrosourea, MNNG: $N$-methey- $N$ '-nitro- $N$-nitrosoguanidine, Br: Brucella broth. 
Table 2 Gastric Carcinogenesis in Helicobacter pylori-infected Mongolian Gerbils

\begin{tabular}{|c|c|c|c|c|c|c|c|}
\hline Author & Year & Strain & cagA gene & $\begin{array}{l}\text { Vacuolating } \\
\text { cytotoxin }\end{array}$ & $\begin{array}{l}\text { Incidence of } \\
\text { cancer }(\%)\end{array}$ & $\begin{array}{l}\text { Duration of } \\
\text { experiment }\end{array}$ & $\begin{array}{l}\text { Histological type of } \\
\text { carcinoma }\end{array}$ \\
\hline Watanabe et al. & 1998 & $\mathrm{TN}_{2} \mathrm{GF}^{*}{ }^{*}$ & + & + & $10 / 27(37)$ & 62 weeks & $\begin{array}{l}\text { well differentiated } \\
\text { adenocarcinoma }\end{array}$ \\
\hline Honda et al. & 1998 & ATCC $43504^{\dagger}$ & + & + & $2 / 5(40)$ & 72 weeks & $\begin{array}{l}\text { well differentiated } \\
\text { adenocarcinoma }\end{array}$ \\
\hline
\end{tabular}

*H. pylori isolated from patient with gastric ulcer. ${ }^{\dagger}$ Type strain.

uated at 40 weeks after an experiment in which 7 week old animals were inoculated with $H$. pylori (ATCC43504) and given a range of doses of MNU before or after inoculation. In this report, the most interesting finding is that only the groups of the animals infected with $H$. pylori together with administration of MNU developed gastric cancers; more specifically, they developed two different types of adenocarcinoma: intestinal and diffuse types, at significantly higher frequencies than did animals given single treatment of either $H$. pylori or the carcinogen. These interesting experimental results support the results so far obtained in large-scale epidemiological investigations. ${ }^{18}$

The group inoculated with $H$. pylori after being given MNU showed a distinctive initiation-promotion effect, whereas the group to which MNU was given after inoculation with $H$. pylori appeared to demonstrate the simultaneous action of these two factors, with $H$. pylori acting as a co-initiator. No gastric carcinoma was found within 40 weeks of $H$. pylori infection alone.

In 1999, two successful experiments on carcinogenicity were reported in which $H$. pylori (ATCC43504) was studied in combination with MNNG. Tokieda et al. ${ }^{16}$ conducted a study in which 5 -week-old Mongolian gerbils were inoculated with $H$. pylori and orally given MNNG at $50 \mathrm{~g} / \mathrm{ml}$ for 20 weeks for comparison against animals administered with MNNG alone. At 52 weeks after initiation of the study, the stomachs were excised for histopathological analysis. The group treated with MNNG and $H$. pylori developed gastric carcinoma at a significantly higher frequency than the group treated with MNNG alone. In addition, when cell proliferation of the pyloric mucosa was evaluated using a labeling index of 5-bromo-2'-deoxyuridine (BrdU), as a lebeling index, cell proliferation was revealed to have markedly accelerated in those animals infected with $H$. pylori. This result suggests the possibility of explaining the link between $H$. pylori infection and early events in gastric carcinogenesis. Of special interest in this study is the discovery that administration of MNNG reduced the infection rate of $H$. pylori with the lapse of time, due to the likelihood of MNNG showing low-level $(200 \mu \mathrm{g} / \mathrm{ml})$ antibacterial activity against $H$. pylori. ${ }^{16}$ It is also of interest that $H$. pylori-free animals did not develop gastric carcinoma. This is an important finding implicating $H$. pylori infection as one of the major factors in the development of gastric carcinoma. Again in this experiment, no carcinoma was found within 52 weeks in those animals with $H$. pylori infection alone.

Shimizu et al. ${ }^{17}$ reported similar results, in which $H$. pylori infection was confirmed to enhance gastric carcinogenesis in Mongolian gerbils treated with MNNG. Furthermore, giving MNNG to infected gerbils decreased the infection rate for $H$. pylori with concomitant reduction in the promotion effect.

Although it has been reported by Sugiyama ${ }^{15}$ that $H$. pylori can perstently colonize the stomach of MNUtreated Mongolian gerbils, it is interesting that the two studies by Tokieda ${ }^{16}$ and Shimizu ${ }^{17}$ report that MNNG administration eradicates $H$. pylori infection, resulting in a reduction of its carcinogenic effects on the glandular stomach.

\section{Carcinogenic Experiments with $\boldsymbol{H}$. pylori Infection Alone}

Two experimental studies attempted to confirm prior epidemiological studies that have demonstrated an association between $H$. pylori infection and gastric carcinogenesis in humans using Mongolian gerbils chronically infected with this bacterium (Table 2) ${ }^{19,20}$ Both studies confirmed gastric carcinogenesis resulting from H. pylori infection alone, and were the first papers to fulfil Koch's postulates concerning $H$. pylori infection and gastric carcinoma.

Watanabe et al. ${ }^{19}$ used $H$. pylori isolated from patients with gastric ulcer (TN2GF4), and Honda et al. ${ }^{20}$ used ATCC43504 (type strain), both of which were inoculated into 5-week old SPF Mongolian gerbils. The results showed that $37 \%$ (10 out of 27 ) of the animals in the former study developed well-differentiated adenocarcinoma at 62 weeks after inoculation, whereas $40 \%$ ( 2 out of 5 ) of the animals in the latter study developed well-differentiated adenocarcinoma at 72 weeks after 
inoculation. Both of these strains contained cytotoxinassociated gene $(\operatorname{cag} A)$ and produced vacuolating cytotoxins. Presence and $H$. pylori infection was confirmed throughout the experiments.

These two studies provided similar findings on sequential histopathological changes. Chronic active gastritis developed initially in the lesser curvature of the antrum, accompanied by infiltration of polymorphonuclear and mononuclear cells, extending eventually to the fundic regions. In our study, ${ }^{20}$ developments including gastric ulcer, atrophic gastritis, and focal intestinal metaplasia began to emerge about six months after $H$. pylori inoculation. These findings also extended to the fundic regions and greater curvatures in association with inflammatory cell infiltration. Intestinal metaplasia then became severe and associated with dysplasia at 12 and 18 months after $H$. pylori inoculation. At 18 months after the sequential histopathological changes in the gastric mucosa, welldifferentiated gastric adenocarcinoma was found in the pyloric regions of the Mongolian gerbil.

Watanabe et al. ${ }^{19}$ also reported that all gastric cancers developed in the pyloric regions and consisted of well-differentiated intestinal-type epithelium, corresponding to intestinal-type adenocarcinoma in humans. All the gastric cancers reported in the two experimental studies developed in the pyloric regions and were of a well-differentiated type. Furthermore, sequential histopathological changes leading to carcinogenesis of the gastric mucosa were found to be common to the two studies, and very closely resembled the histopathological changes in human gastric mucosa caused by $H$. pylori infection.

The most important study yet to be carried out will be to extrapolate the sequential histopathological changes found in Mongolian gerbils to lesions in the human gastric mucosa, and to address the possible mechanism through which $H$. pylori infection may result in a predisposition to gastric carcinoma. However, a slight discrepancy was found between these studies regarding intestinal metaplasia and location of gastric carcinoma, and further studies are required to clarify the association between gastric carcinogenesis and intestinal metaplasia. Until now, there have been no reports of animal studies which confirm the development of diffuse type gastric adeno-carcinoma as a result of $H$. pylori infection alone, thus necessitating further studies.

Table 3 shows our tentative opinions on p53 and $H$. pylori infection in human, Japanese monkeys and Mongolian gerbils. ${ }^{21}$ In humans and Japanese monkeys both p53 immunostaining and point mutations were observed in those with $H$. pylori infection.

It is quite difficult to explain why gastric carcinoma occurs so easily in Mongolian gerbils when there is so
Table 3 Relation Between p53 and $H$. pylori and Gastric Cancer (tentative opinion)

\begin{tabular}{lccc}
\hline \hline Condition & Human & $\begin{array}{l}\text { Japanese } \\
\text { monkey }\end{array}$ & $\begin{array}{l}\text { Mongolian } \\
\text { gerbil }\end{array}$ \\
\hline $\begin{array}{l}\text { H. pylori and p53 } \\
\text { overexpression (histology) }\end{array}$ & 0 & 0 & $\Delta^{\mathrm{a}}$ \\
$\begin{array}{l}\text { H. pylori and p53 point } \\
\text { mutation (exons 5-8) }\end{array}$ & 0 & 0 & $\times$ \\
H. pylori and gastric cancer & $\Delta^{\mathrm{b}}$ & $\times$ & 0 \\
\hline
\end{tabular}

"The p53 overexpression was observed only in gastric cancer. ${ }^{b}$ Not proven by interventional study. Circles: strong evidence, triangles: weak evidence, crosses: no evidence.

little evidence of p53 mutations in $H$. pylori infected gastric mucosa. It possibly suggest that the other tumorrelated genes are involved in the carcinogenesis in this animal.

\section{Conclusions}

Experiments developed in Japan using Japanese Monkeys and Mongolian gerbils have demonstrated that $H$. pylori infection is clearly responsible for the progression of atrophic gastritis and gastric carcinogenesis, and provide important confirmation of the statements issued by IARC/WHO. It will be of critical importance to extrapolate the sequential histopathological changes found in the Japanese monkey and Mongolian gerbil to lesions in the human gastric mucosa, since this model is proven to provide important pointers for the study of the mechanism of gastric carcinogenesis as a result of $H$. pylori infection. The most important study yet to be performed is extrapolation of p53 mutations and histopathological changes in animal model with lesions common to the human gastric mucosa.

\section{References}

1. Parsonnet J, Freedman GD, Vandersteen DP, Chang Y, Vogelman JH, Orentreich N, Sibley RK: Helicobacter pylori infection and risk of gastric carcinoma. N Engl J Med 1991; 325: 11271131

2. Nomura A, Stemmerman GN, Chyou P-H, Kato I, Perez PG, Blaser MJ: Helicobacter pylori infection and gastric cancer among Japanese Americans in Hawaii. N Engl J Med 1991; 325: $1132-1136$

3. Forman D, Newell DG, Fullerton F, Yarnell JWG, Stacey AR, Wald N, Sitas F: Association between infection with Helicobacter pylori and risk of gastric cancer: evidence from a prospective investigation. BMJ 1991; 302: 1302-1305

4. International Agency for Research on Cancer: Infection with Schistosomes, Liver Flukes and Helicobacter pylori. IARC Monographs on the Evaluation of Carcinogenic Risks to Humans. 1994; 61: 218-220 
5. Shuto R, Fujioka T, Kubota T, Nasu M: Experimental gastritis induced by Helicobacter pylori in Japanese monkeys. Infect Immun 1993; 61: 933-939

6. Fujioka T, Kodama R, Honda S, Guei-Hua G, Nishizono A Nasu M: Long-term sequelae of experimental gastritis with Helicobacter pylori: A 5-year follow-up study. J Clin Gastroenterol 1997; 25: S8-S12

7. Kodama M, Fujioka T, Kodama R, Takahashi K, Kubota T, Murakami K, Nasu M: p53 expression in gastric mucosa with Helicobacter pylori infection. J Gastroenterol Hepatol 1998; 13: 215-219

8. Oda T, Murakami K, Nishizono A, Kodama M, Nasu M, Fujioka $\mathrm{T}$ : Long-term Helicobacter pylori infection in Japanese monkeys induces atrophic gastritis and accumulation of mutations in the p53 tumor suppressor gene. Helicobacter 2002; 7: 143-151

9. Hirayama F, Takagi S, Kusuhara H, Iwao E, Yokoyama Y, Ikeda Y: Induction of gastric ulcer and intestinal metaplasia in the gastric mucosa of Mongolian gerbils infected with Helicobacter pylori. J Gastroenterol 1996; 31: 755-757

10. Honda $S$, Fujioka T, Tokieda M, Gotoh T, Nishizono A, Nasu $\mathrm{M}$ : Gastric ulcer, atrophic gastritis, and intestinal metaplasia caused by Helicobacter pylori infection in Mongolian gerbils. Scand J Gastroenterol 1998; 33: 454-460

11. Ikeno $T$, Ota $H$, Sugiyama A, Ishida K, Katsuyama T, Genta RM, Kawasaki S: Helicobacter pylori-induced chronic active gastritis, intestinal metaplasia, and gastric ulcer in Mongolian gerbils. Am J Path 1999; 154: 951-960

12. Tatematsu M, Yamamoto M, Shimizu N, Yoshikawa A, Fukumi H, Kaminishi M, Oohara T, Sugiyama A, Ikeno T: Induction of glandular stomach cancers in Helicobacter pylori-sensitive Mongolian gerbils treated with $N$-methyl- $N$-nitrosourea and $N$ methyl- $N$-nitro- $N^{\prime}$-nitrosoguanidine in drinking water. Jpn $\mathbf{J}$ Cancer Res 1998; 89: 97-104
13. Fox JG, Wishnok JS, Murphy JC, Tannenbaum SR, Correa P: MNNG-induced gastric carcinoma in ferrets infected with Helicobacter mustelae. Carcinogenesis 1993; 14: 1957-1961

14. Fox JG, Dangler CA, Sager W, Borkowski R, Gliatto JM: Helicobacter mustelae assiciated gastric adenocarcinoma in ferrets (Mustela outorius furo). Vet Pathol 1997; 34: 225-229

15. Sugiyama A, Maruta F, Ikeno T, Ishida K, Kawasaki S, Katsuyama T, Shimizu N, Tatematsu M: Helicobacter pylori infection enhances $N$-Methyl- $N$-nitrosourea-induced stomach carcinogenesis in the Mongolian gerbil. Cancer Res 1998; 58: 20672069

16. Tokieda M, Honda S, Fujioka T, Nasu M: Effect of Helicobacter pylori infection on the $N$-methyl- $N^{\prime}$-nitro- $N$-nitrosoguanidineinduced gastric carcinogenesis in Mongolian gerbils. Carcinogenesis $1999 ; 20: 1261-1266$

17. Shimizu N, Inada $K$, Nakanishi $H$, Tsukamoto $T$, Ikehara $Y$, Kaminishi M, Kuramoto S, Sugiyama A, Katsuyama T, Tatematsu M: Helicobacter pylori infection enhances glandular stomach carcinogenesis in Mongolian gerbils treated with chemical carcinogens. Carcinogenesis 1999; 20: 669-676

18. Huang JQ, Sridhar S, Chen Y, Hunt RH: Meta-analysis of the relationship between Helicobacter pylori seropositivity and gastric cancer. Gastroenterology 1998: 114: 1169-79

19. Watanabe T, Tada M, Nagai H, Sasaki S, Nakao M: Helicobacter pylori infection induces gastric cancer in Mongolian gerbils. Gastroenterology 1998; 115: 642-648

20. Honda S, Fujioka T, Tokieda M, Satoh R, Nishizono A, Nasu M: Development of Helicobacter pylori-induced gastric carcinoma in Mongolian gerbils. Cancer Res 1998; 58: 4255-4259

21. Murakami K, Fujioka T, Kodama M, Honda S, Okimoto T, Oda T, Nishizono A, Sato R, Kubota T, Kagawa J, Nasu M: Analysis of 553 mutations and Helicobacter pylori infection in human and animal model. J Gastroenterol 2002; 37(suppl 13): 1-5 